\title{
THE 'TOOTH' OF THE DOG THAT BIT HIM
}

\author{
D. P. CONLAN
}

Case report. A 14-year-old schoolboy was bitten by an Alsatian dog, sustaining a puncture wound over the dorsal surface of the terminal interphalangeal joint of the right little finger. Primary closure of the wound was performed without wound toilet and no antibiotics were given. One week later the wound was infected and flucloxacillin was prescribed. He continued to have considerable pain, swelling and discharge from the wound; a swab was therefore taken and the antibiotic was changed to Augmentin.

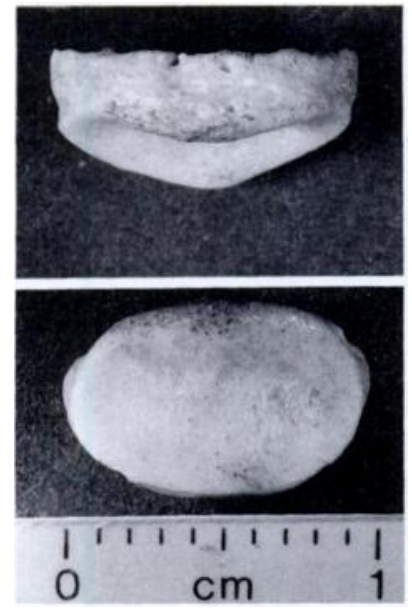

Fig. 1

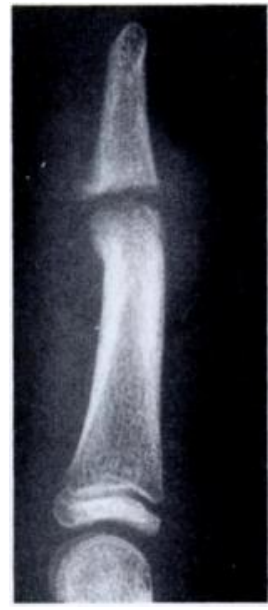

Fig. 2
At first review in the orthopaedic department four weeks after the injury, the patient reported that the infection had suddenly improved when the "dog's tooth had come out of the wound". On examination there was mild erythema around the distal interphalangeal joint of the affected finger, but no discharge; the joint was extremely stiff. Closer examination of the 'dog's tooth'

D. P. Conlan, FRCS, FRCS (Ed), Lecturer in Orthopaedics University Department of Orthopaedics, Glenfield General Hospital, Groby Road, Leicester LE3 9QP, England.

(C) 1989 British Editorial Society of Bone and Joint Surgery 0301-620X/89/5R $40 \$ 2.00$

J Bone Joint Surg [Br] 1989;71-B:858. revealed it to be an epiphyseal plate (Fig. 1) and a radiograph confirmed loss of the epiphyseal plate, narrowing of the joint space and bone erosion (Fig. 2).

As there was no growth from the recent culture, antibiotic therapy was continued with Augmentin and the finger was splinted in the position of function. Following this the infection remained quiescent and the joint fused in $15^{\circ}$ of flexion.

Discussion. Cases of septic arthritis and osteomyelitis of the hand following neglected bites are not uncommon, but extrusion of a phalangeal epiphysis as a sequestrum has not, as far as we know, previously been reported.

Although only $2 \%$ to $5 \%$ of all animal bite wounds become infected, as many as $30 \%$ of such wounds of the hand do so (Callaham 1980). With dog and cat bites attention has recently been focused on Pasteurella multicocida, as the most commonly isolated infecting organism (Arons, Fernando and Polayes 1982). Prophylactic antibiotics appear to reduce the incidence of infection (Thomas and Buntine 1987) and a cephalosporin or, for animal bites, penicillin (the drug of choice for Pasteurella multicocida) is recommended. However, cases of penicillin resistance have been reported, possibly because of the veterinary practice of prescribing antibiotics (Chapple and Fraser 1986).

This present case report emphasises the potentially serious consequences of neglected animal bites of the hand. It is suggested that wound toilet followed by prophylactic antibiotic therapy should always be performed.

The author would like to thank Mr J. H. Newman of British Royal Infirmary for permitting this case to be reported.

No benefits in any form have been received or will be received from a commercial party related directly or indirectly to the subject of this article.

\section{REFERENCES}

Arons MS, Fernando L, Polayes IM. Pasteurella multocida: the major cause of hand infections following domestic animal bites. $J$ Hand Surg [ Am] 1982:7:47-52.

Callaham M. Dog bite wounds. JAMA 1980;244:2327-8.

Chapple CR, Fraser AN. Pasteurella multocida wound infections: a commonly unrecognised problem in the casualty department. Injury 1986;17:410-1.

Thomas PR, Buntine JA. Man's best friend?: a review of the Austin Hospital's experience with dog bites. Med J Aust 1987;147:536-40. 